\title{
Explicitly Privacy-Aware Space Usage Analysis
}

\author{
Sanjiv S. Jha \\ Institute of Computer Science \\ University of St. Gallen \\ St.Gallen, Switzerland \\ sanjiv.jha@unisg.ch
}

\author{
Simon Mayer \\ Institute of Computer Science \\ University of St. Gallen \\ St.Gallen, Switzerland \\ simon.mayer@unisg.ch
}

\author{
Tanja Schneider \\ Technology Studies \\ School of Humanities and Social Sciences \\ University of St. Gallen \\ St.Gallen, Switzerland \\ tanja.schneider@unisg.ch
}

\begin{abstract}
Surveillance in private and public spaces provides observers with information that can enhance protection and efficiency but usually infringes upon the privacy of the individuals and groups. These informational privacy risks are centered on users' perceived and design-induced threats. They cannot be removed completely but can be minimized using suitable anonymization techniques. To minimize the users' informational privacy threats, we designed a privacy-aware surveillance system that gives the users leverage over the anonymization filters, to physically adjust the opaqueness of the camera lens used in the prototype according to their privacy requirements. We implement our prototype in the context of office space surveillance, where the proposed solution considers privacy requirements in such environments to improve users' trust in the surveillance system and reduce their privacy concerns.
\end{abstract}

Index Terms-privacy, perceived privacy, surveillance, camerabased, office-space, privacy-aware

\section{INTRODUCTION}

We share our personal details in the modern (digital) world unknowingly with a click of a button or simply by walking in the field of view of a CCTV camera, presuming that the gathered information will not be brought into the public eye without any legal justification. However, our privacy becomes prone to intrusions as soon as we reveal our personal details to other parties or if others have the means to identify individuals (e.g., using facial recognition technology). Moreover, many examples of non-consented information sellers including social media and insurance companies are listed in [1], also researchers emphasize the severity of existing data privacy threats posed in a digital society [2].

Camera-based surveillance requires individuals to consent to the monitoring - i.e., a certain degree of explicit acceptance of the surveillance equipment is required. Also, surveillance systems should be proofed to reduce the risk of privacy infringement [4], which can for instance be achieved by employing visual anonymization techniques. While detection of individuals is simplified with current deep learning systems [6], applying visual modifications to the input (e.g., added noise) may confuse the neural network into making wrong predictions [7]. Besides, many of the available tracking technologies for real-time behavioral analysis (e.g., occupancy detection and in-store analytics [8]) are resourceand maintenance-heavy because of accuracy and correctness concerns. However, if fine details of the gathered data are not of concern for observers (e.g., if the application itself does not require user identification in the first place), it is possible to reduce the accuracy of the gathered data while minimizing costs, for instance by reducing the number of sensors and using multi-modal data fusion approaches [5].

With this article, we aim to provide a way to preserve the privacy of workers in an office space that is being monitored to enable space usage analysis. We focus on video surveillance and video privacy protection mechanisms and propose a privacy-aware surveillance system that aims to minimize the aforementioned privacy infringement risks in video surveillance systems. The ultimate goal of developing a privacyaware space usage analysis system is to prevent the identification of office workers, which would support the uptake of the system. The use of camera-based surveillance implicitly carries the risk of informational privacy violation [10]. This risk is not only introduced through human errors or system security flaws but it can also be due to additional information (faces, behaviors and, surroundings, etc. of individuals in the field of view) that is captured by a camera module compared to other sensor/tracking modules (e.g., motion sensors, beaconing systems, etc.) used in surveillance. Since the implicit risk of using video surveillance derives from the use of image frames in the analysis of gathered video footage, we can ground the origin of such privacy threats to the design of the setup similar to cryptanalysis attack risks in cryptography protocols [13].

In our application, we can afford to lose fine details of the gathered images, because the project aims to evaluate an office space usage (see Section II) through a prototype, rather than the identification of individuals in that space. Hence we suggest a privacy-aware framework in the same line as the privacy-in-surveillance systems achieved by incorporating noise. Importantly, we separate video surveillance privacy concerns into two types, design-induced informational privacy risk and perceived informational privacy risk. We define design-induced (DI) informational privacy risk as the likelihood of the above-mentioned privacy intrusions happening to individuals through visual data collection without their consent, some of the examples (e.g., misuse and abuse of sensitive video data) are explained in [10]. Perceived privacy risk, on the other hand, can be described as the level at which an individual believes that a system might have negative consequences for their informational privacy [14], [15]. Our proposed system aims to improve camera-based surveillance systems regarding individuals' perceived and DI privacy risks; 
we address the following research questions:

1) How can we implement a camera-based privacypreserving surveillance system to detect space usage?

2) How does this privacy-preserving system respond to various situations that could compromise users' perceived and $D I$ privacy?

To answer these research questions, we implemented a prototype based on a case study elaborated in the following section. On the one hand, this prototype addresses the problem of DI privacy risks in camera-based surveillance systems to answer our first research question using an image blurring process; and on the other hand, it offers a way to adapt the system to the evolving privacy requirements of the office environment using an external, physical, blurring approach. Using the physical blurring approach, users can adjust the visibility through the camera lens, by attaching external camera lens covers of different opaqueness levels. This is to reduce the DI risks and, perhaps, also the perceived risks to their privacy. We use $\mathrm{WiFi}$ and $\mathrm{QR}$-code technology to enable users to access the (blurred) real-time video feed captured via the camera to improve their users' confidence in the device (the corresponding implementation can be seen in Figure 1a and Figure 1b). A sociological, qualitative research study focused on the impact of such a program on those being under surveillance is conducted in parallel to our deployment.

The following section elaborates on the case study used to substantiate the internal and physical blur mechanisms in office spaces. Subsequently, we discuss our privacy-aware surveillance system in more detail in Section III and present results from a performance evaluation that we conducted in our office environment in Section IV. We position our contribution concerning related work in Section V and discuss our planned future research on top of this system in Section VI.

\section{Case Study (Furniture Usage Analyzation)}

Our privacy-aware surveillance system is focused on an office environment that is shared by many employees. In this context, employers want to rearrange and optimize the area used by furniture to maximize space use and recurrent contact among employees. For instance, employers would like to understand which of the deployed furniture items are used rarely and can thus be removed. While this can in principle is achieved through simple monitoring, the challenge is that employers would like to collect information about the use of office furniture without infringing upon the office worker's (DI as well as perceived) privacy. Furthermore, two types of furniture usage are relevant in this case, we term them as warm and cold usage: If any motion is observed in the recorded changes then they are referred to as a moving (warm) usage, with active ongoing use, whereas, when there is no continuous movement but the furniture item is used passively then these changes are referred to as a static (cold) usage. Both of these changes carry meaning concerning the use of a furniture item and influence the optimization potential - for instance, a whiteboard or chest might be deemed necessary even though only a few active interactions with it are recorded if these interactions happen regularly (i.e., a lot of cold use compared to warm use).

Given a system that can perform this type of monitoring without undermining the perceived and DI privacy of the users of a space, heat maps, usage reports, and annotated video streams can be created to support the optimization of the office environment. All these three outputs will help the employers to understand the systems' decision (warm usage, cold usage and, room occupancy) better; and they could support furniture suppliers in updating their businesses to a more service-driven business model. Finally, since our use case is based in a public environment, it is crucial to consider the perceived and DI privacy threats of the office workers in different room setups and with different levels of privacy requirements.

\section{PRIVACY-AWARE SURVEILlanCE PROTOTyPE}

We present a novel approach to explicitly preserve privacy in surveillance systems used for usage type detection taking into account all the requirements of the situation. Since the case study of this prototype is focused on furniture usage, the system can distort the footage of office workers to preserve their privacy. Hence, we use blurring mechanisms (internal/external) to achieve privacy preservation in such a system. For office space surveillance, the proposed prototype can be configured to function for either of the blurring techniques (software blur as well as physical blur) mentioned in Section I. The prototype uses a physical lens cover (the corresponding prototype can be seen in Figure 1b) for reducing the perceived privacy risks among the office workers while preserving their DI privacy simultaneously. The cover can be changed to less or more opaqueness based on the situation. This adaptable nature of the setup to achieve different levels of privacy requirements makes the system more privacy-aware than just privacy-preserving. The system can sense the degree of blurriness generated by a certain lens overlay and adjust the necessary change-detection parameters (i.e., threshold value, discussed in Section III-B4) to preserve the DI privacy of the users. In the following, we introduce the privacy-aware surveillance system we developed for detecting the type of furniture used in our target environment.

\section{A. Setup}

This prototype implementation addresses our first research question by using either of the introduced blurring mechanisms in camera-based furniture usage type detection systems. To implement this setup, we used Raspberry $\mathrm{Pi}^{1}$ devices with $\mathrm{Pi}$ Cameras and fish-eye lenses. As discussed in the introduction section, to provide comfort and perceived privacy to the employees, a small size (to be seemingly unnoticeable in the environment) of the setup is desired. Additionally, adequate performance is required (more processing power than an Arduino $\mathrm{Uno}^{2}$ for the post-processing phase) which is why we selected RPi and Pi Camera modules. Figure 1a shows the

\footnotetext{
${ }^{1}$ https://www.raspberrypi.org

${ }^{2}$ https://www.arduino.cc/en/Guide/ArduinoUno
} 
physical prototype assembly of the prototype, where a fisheye lens is mounted on top of the Pi Camera and RPi setup and connected to the power supply. The setup can be powered using a Li-ion battery for flexibility in short term physical system installations. Because the current system only detects movements locally in real-time and does not send the data to other data processing systems on an ongoing basis. However, for extended period deployments, mains power is required. A WiFi connection is only used to maintain the system using Virtual Network Computing (VNC) or for optional data transmission to 3rd party applications. The software is implemented using Python 3 as a programming language with the OpenCV $2^{3}$ library for image segmentation and image augmentation. The application is deployed on the Raspberry $\mathrm{Pi} \mathrm{OS}^{4}$ and allows a VNC connection for real-time frame display and system checks.

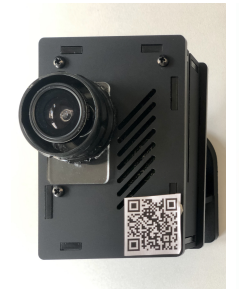

(a)

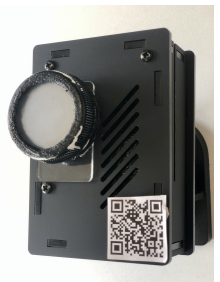

(b)
Fig. 1. Prototype without the lens cover (a) and with lens cover (b).

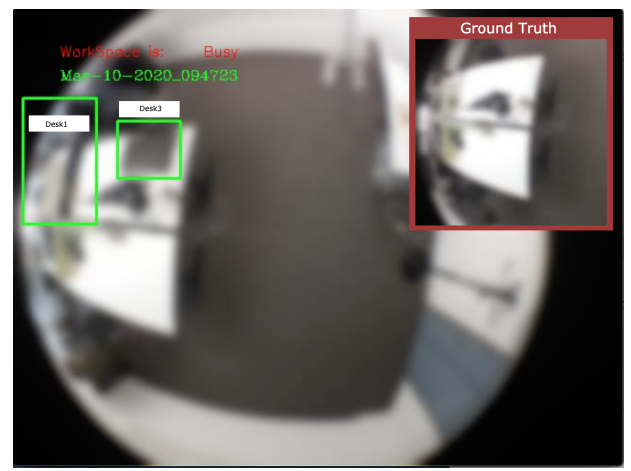

Fig. 2. Areas with detected changes.

\section{B. System Workflow}

The prototype starts collecting the surveillance footage for usage analysis as soon as its bootup process is completed. When the user assigned duration of surveillance has elapsed, all the changes are registered into a changing array. The functions responsible for delivering the analysis results then take the changes from the environment as input and tests them against a region of interest (RoI), i.e., the specified dimensions for the area occupied by the furniture, wall, etc. under surveillance, as can be seen in Figure 3. It also checks for continuity

\footnotetext{
${ }^{3}$ https://opencv.org/

${ }^{4}$ https://www.raspberrypi.org/downloads/raspberry-pi-os/
}

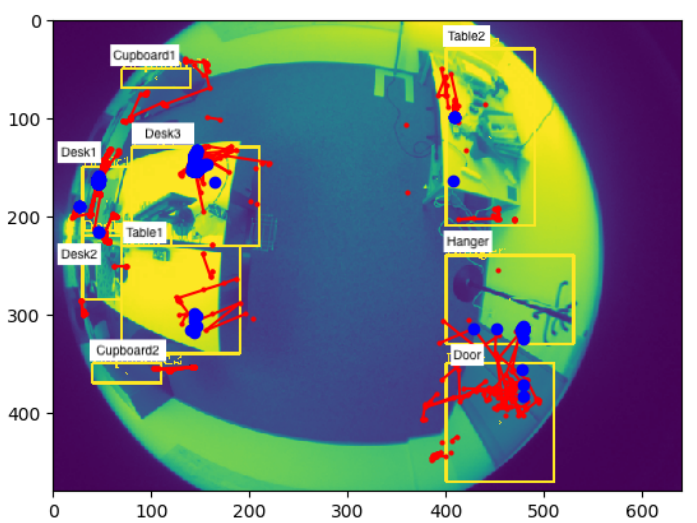

Fig. 3. Output Graph, where the blue overlays denote cold usage and red overlays denote warm usage of the area or furniture item.

TABLE I

FuRNITURE UsAGE ANALYZATION RESUlts

\begin{tabular}{|l|l|l|l|l|}
\hline $\begin{array}{l}\text { Furniture } \\
\text { Type }\end{array}$ & $\begin{array}{l}\text { Usage } \\
\text { Count }\end{array}$ & $\begin{array}{l}\text { Total } \\
\text { Changes }\end{array}$ & $\begin{array}{l}\text { Usage Per- } \\
\text { centage }\end{array}$ & $\begin{array}{l}\text { Usage } \\
\text { Type }\end{array}$ \\
\hline Cupboard1 & 0 & 16215 & 0 & cold \\
Cupboard2 & 0 & 16215 & 0 & cold \\
Desk1 & 11936 & 16215 & 73.61 & cold \\
Desk2 & 1 & 16215 & 0.01 & cold \\
Desk3 & 14889 & 16215 & 91.82 & cold \\
Door & 3 & 16215 & 0.02 & cold \\
Hanger & 8936 & 16215 & 55.11 & cold \\
Table1 & 7 & 16215 & 0.04 & cold \\
Table2 & 6 & 16215 & 0.04 & cold \\
\hline Cupboard1 & 1461 & 16215 & 9.01 & warm \\
Cupboard2 & 48 & 16215 & 0.3 & warm \\
Desk1 & 12822 & 16215 & 79.07 & warm \\
Desk2 & 741 & 16215 & 4.57 & warm \\
Desk3 & 2335 & 16215 & 14.4 & warm \\
Door & 142 & 16215 & 0.88 & warm \\
Hanger & 94 & 16215 & 0.58 & warm \\
Table1 & 379 & 16215 & 2.34 & warm \\
Table2 & 1138 & 16215 & 7.02 & warm \\
\hline
\end{tabular}

among the changes gathered to distinguish between the two types of changes the system aims to detect (i.e., warm and cold changes). When these changes are identified in the userdefined RoI, they are plotted on a clear layout that is supplied by the user at the start of the process. One of the system's core functionality is detecting motion in the area to declare that the area is free or busy, shown in Figure 2. The process flow overview of our system is depicted in Figure 4. This process flow is common for both the privacy-preservation mechanisms mentioned above (i.e., software blur and physical blur). With a software blur, the system however additionally applies a blur filter on the captured frames before change-detection, unlike the physical blur mechanism.

1) Software blur: With the software blur method, the camera lens is visible to the users (the corresponding prototype can be seen in Figure 1a), and to preserve the privacy of users, our system adds a software blur filter. The system sets this filter's size parameter to a certain value (e.g., Gaussian blur kernel size 31 X 31) that provides a level of anonymization to the surveillance footage, so the captured frames lose the fine 
details necessary to identify a person. To protect privacy, all captured frames are first intentionally blurred by the system before the motion detection procedure starts.

Since the camera lens is exposed to users when only employing software blurring, this method reduces DI privacy risks but might increase users' perceived privacy risk [19]. Using internally-blurred methods alone is thus not adequate for reducing the overall privacy risks of employees [20], [21] without any camera hiding mechanisms because of the visible lens. We, therefore, suggest a physical lens overlay to add distortion to the resulting images, which might be an acceptable solution to users. Hence, we furthermore introduce an physical blurring mechanism.

2) Physical blur: This physical blur method uses a physical lens cover to lower the perceived privacy risks of the users. We hypothesize that the lens covers used in the physical blur mechanism, obfuscate the camera image enough to preserve individuals' privacy while at the same time supporting the acceptance of our surveillance system. This overlay (i.e., a semi-transparent lens cover) produces a blurred image of the monitored area which is then processed for change-detection. For example, if hiding details of a confidential meeting is important, then high blurriness of the images can be achieved by using a highly opaque lens cover. However, a more transparent lens cover can be used in situations where less blur is acceptable to users of the system. In the case of the physical blur, we take advantage of the obscurity provided by the physical lens cover, and no software blur-filter is added to the images because the images captured are already blurred.

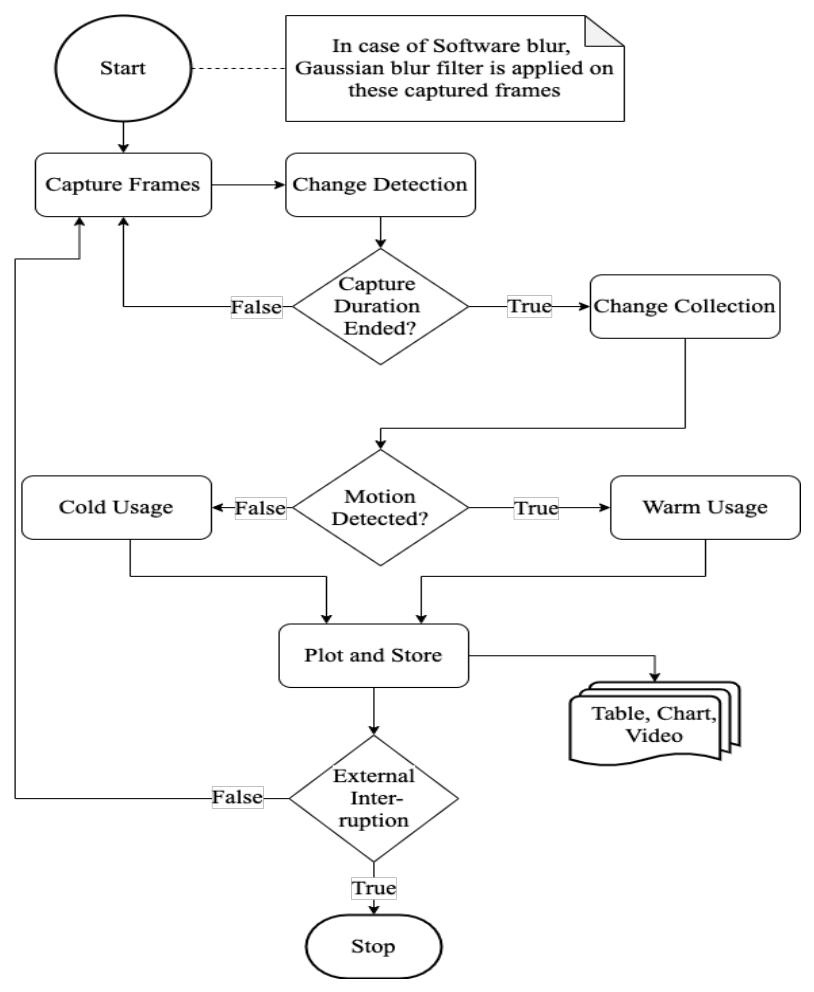

Fig. 4. Process Flow Diagram.
3) change-detection: For change-detection in the field of view, our prototype uses one of the Background Subtraction (BS) approaches. BS is a method that distinguishes the foreground from the background to detect changes in the pixel values caused by moving objects [16]. Our assumption here is that the moving objects under the field of view are only human beings. Since the blurred images lose important feature details, it is difficult to detect people using blurred images, and it is also not our target. Some of the benefits and drawbacks of the BS method are shown in [17]. For computational efficiency and to optimize the real-time frame rate during the surveillance, we have chosen the basic BS method [18] where the background model is made up of a single frame for the entire surveillance period.

4) System Operation: The system takes the first picture of the environment at the start and sets it as a reference frame (RF). If the cumulative brightness of the RF is not adequate compared to the user-defined threshold, the device goes to sleep for 15 minutes and wakes up again to check if the room is bright enough to continue the monitoring mission. Before taking the difference with the RF, each frame is intentionally blurred. The difference (Delta) is compared with a threshold value between the blurred gray images ( $R F$ - new frame), this technique is also known as Frame-Differencing (FD) [22]. If this Delta is higher than the threshold value, a detection will be assigned to the new frame, otherwise the system will reject this frame and look for a movement in the next one. Each time a movement is detected in a frame, it is stored by the system in an array for detection of the type of use in post processing. Figure 2 shows the reference image or ground truth (GT) and the change-detection in a new frame. It also shows that the room was free in the GT and detected busy in the new frame. Because the height of the furniture item Desk 1 has changed and someone was using Desk3 to keep something on it.

In post-processing, a motion-detection function is applied to the change array. This function decides whether or not the changes gathered to show a certain continuity. If no continuity is found between the changes then they are checked for a repeated number of occurrences. The repeated occurrence of a change in the array hints of cold usage. Otherwise, if there is any continuity found in the changes then those are tagged as warm usage. These modifications are then reported and presented to the observers as an output. The entire usage analysis process never works with unblurred frames, which ensures the end-to-end obscurity of sensitive data. After monitoring the area for the desired period, the RPi camera stops capturing the footage and starts analyzing the recorded changes. Tabular information depicted in Table I presents types of usages reported(either warm or cold), usage percentage (the ratio of the number of detection in RoI and the total number of change-detection), and the total number of changes recorded via the surveillance. The system creates a visual output (i.e., heat map) as well that can be seen in Figure 3. Here the blue circles denote cold usage, red lines are the warm usage of the area or furniture item, and the RoIs are drawn using yellow rectangles. 


\section{Motion Detection}

The distance between consecutive changes within a range determines whether or not motion was present. For this purpose, our system calculates the Euclidean distance between consecutive changes. For example, an Euclidean distance $D(A, B)$ between consecutive change centroids $A\left(a^{x}, a^{y}\right)$ and $B\left(b^{x}, b^{y}\right)$, assuming that there are many changes consecutively occurring in the RoI, is

$$
D(A, B)=\sqrt[2]{\left(a^{x}-b^{x}\right)^{2}+\left(a^{y}-b^{y}\right)^{2}} .
$$

If the distance $D(A, B)$ is greater than the threshold (set between $0-3$ ) then they are considered as a warm usage, otherwise a cold usage. The core idea behind annotating warm and cold usage over a heat map is to generate an awareness of space used and help deduce possible rearrangement of the furniture in the room. This analysis is presumed to be essential for increasing the productive interactions and optimal space utilization measures.

\section{EVALUATION AND DISCUSSION}

The system evaluation is done based on the case study explained in Section II. We limit the area under surveillance to be adequately lit and an optimal condition for evaluation (i.e., an controlled environment that would give less lighting fluctuations), to identify the optimal solution found through the prototype.

To address our first research question, the system is evaluated employing an expert evaluation method in which the accuracy of furniture space occupancy detection was checked for a random selection of frames. The accuracy metrics for change-detection were employed based on the knowledge of human experts to identify cold and warm usage in the frames. We ran the surveillance system for one week in an office environment under a controlled setup and collected the data in a loop cycle of three hours, i.e., 168 number of samples of the recordings. We randomly selected 50 scenes from 168 samples captured by the system for this evaluation, and then spread them out to frames. The accuracy was measured based on the ratio between the number of correct changes the system detected and the total number of changes in the chosen frames. The expert assessor was asked to select random frames and classify changes irrespective of what has been observed in the system. The experts were asked to continuously refer to the RF and the previous frame for correct identification of changes. We compared both values, the accuracy of the system turned out to be around 80 percent in a controlled setup. The measured precision of correct detection will however vary depending on the environment the device is being tested in.

To answer our second research question, we developed proofs of concept (POC) for both blurring approaches (physical and software). A POC with a physical lens cover can be adapted to different scenarios by changing the lens cover to manipulate the blur level of the camera. This physical control i.e, lens cover, gives more control over the system to the users. The POCs have been deployed in a real-world office environment that will help us assess the system efficiency and the benefits given to office staff regarding perceived privacy using our adaptive lens covers. A parallel sociological study will help us to understand the implication of this setup for office workers and how their perceived privacy is affected using adaptive lens covers. In this setup, we have deployed a few prototypes without any lens covers to rely completely on software blur for privacy-preserving. Hence, the result of this survey would also help us to deduce the acceptance level of the physical blur system over the software blur system by office workers.

As per the case study, the presented solution is not focused on individuals present in the monitored environment, but values employees' privacy while analyzing space and furniture item usage. Since the visible physical lens cover on the camera lens indicates that workers are not being observed through it, we believe that this arrangement maintains a privacy-friendly working environment while still delivering an effective analysis system by appreciating both the DI and perceived privacy of employees. Also, the system can be adapted to detect the level of obscurity of a frame caused by the lens cover. Such a system enhancement with varying levels of opaqueness of lens covers is a first step towards creating a more contextaware monitoring system in the future. A qualitative study on human acceptance and the perceived privacy of the system will allow us to gain in-depth insights from users to inform new concepts and products.

During surveillance and the change-detection, the prototype does not update the background model because retaining the changes compared to the first captured frame is necessary for detecting and maintaining the cold usage. It is crucial for our case study but at the same time, it also causes several falsepositive change-detection due to lighting changes in the environment increases. The gathered footage taken during sunrise and sunset has more false positives (i.e., change detected but there was no real movement in the surrounding, etc.) than the rest of the day: as observed in the current system. The future amelioration of the prototype would focus on statistical background variation modeling to lower the number of falsepositive change-detections.

\section{RELATED WORK}

Not only the DI but also perceived privacy risks are targeted through this article. [9] has stated that the explicit visibility of cameras or surveillance measures makes employees seem worried in the workplace leading to reduced creativity. To address this issue, in our research we seek to reduce the risk of documenting individuals' actions by installing an privacypreserving surveillance system at the room ceiling similar to [11]. Additionally, since the camera has a physical lens cover it may look less intimidating to the employees.

Although it was deduced in a study by [20], [21] that the blur filter alone (software blur) was not successful in providing users with enough perceived privacy in home office setup, current research on maintaining a balance between privacy and analysis efficiency implies that blurring images can be a way to obscure sensitive data for maintaining users' DI 
privacy [23]. [3] explains that customers face a dilemma between maintaining their privacy and benefiting from customized offers and services offered by covetous firms.

In a regional qualitative study carried out by [24], it was noted that individuals are not aware of whether there are any rules to demand clarification on the usage of the gathered data from the observers. Apart from awareness of data usage among users, [11], [12] demonstrated how fragile an individuals' privacy is under state-of-the-art technology-enabled video surveillance. [6] states that any kind of human skin (face, skin tone) under the surveillance footage can behave as a feature that can lead to revealing the provenance of both, the video and people in the footage. There are many possibilities to mitigate some of the video privacy issues by using techniques like distorting images, adding noise, etc. on the identified risk regions in the videos but doesn't target the perceived privacy of the users. The presented solution which employs a physical blurring mechanism can be an effective way to achieve a balance between data collection and the perceived privacy of the users.

\section{CONCLUSION}

We presented an expressly privacy-aware camera-based monitoring system and hypothesize that this should help preserve the perceived as well as the design-induced privacy risk of individuals in a monitored environment. Our prototype, which is used for furniture usage type detection and analysis using software-based as well as physical blurring through a camera lens provides privacy to the people under surveillance, where the physical lenses can be exchanged at run time by users to adapt to situations with different privacy requirements. Our case study of furniture usage analysis demonstrates how this prototype can be deployed and evaluated in an office environment. Although there are some false positive detection results due to natural lighting change and increased blur intensity for user privacy, the system offers good precision and simple handling and maintenance. The presented approach provides overall transparency and more data control to the users. The projects' future scope is to explore deployment opportunities in various sectors (e.g., retail, manufacturing) and to evaluate the impact of our system on human users through a sociological study of human behavior and acceptance of this prototype in an office environment.

\section{ACKNOWLEDGMENT}

The authors gratefully acknowledge Fabrice Guermann and Nora Kueng's efforts and contributions in developing the prototype and concept for this project and article.

\section{REFERENCES}

[1] M. Bashir, C. Hayes, A. D. Lambert, and J. P. Kesan, "Online privacy and informed consent: The dilemma of information asymmetry," Proceedings of the Association for Information Science and Technology, vol. 52, no. 1, pp. 1-10, 2015.

[2] A. Martin, "Digital literacy and the "digital society"," Digital literacies: Concepts, policies and practices, vol. 30, pp. 151-176, 2008.

[3] K. Plangger and M. Montecchi, "Thinking beyond privacy calculus: Investigating reactions to customer surveillance," Journal of Interactive Marketing, vol. 50, pp. 32-44, 2020.
[4] B. Gelbord and G. Roelofsen, "New surveillance techniques raise privacy concerns," Communications of the ACM, vol. 45, no. 11, pp. 23-24, 2002.

[5] K. Akkaya, I. Guvenc, R. Aygun, N. Pala, and A. Kadri, "Iot-based occupancy monitoring techniques for energy-efficient smart buildings," in 2015 IEEE Wireless communications and networking conference workshops $(W C N C W)$, pp. 58-63, IEEE, 2015.

[6] A. Shifa, M. B. Imtiaz, M. N. Asghar, and M. Fleury, "Skin detection and lightweight encryption for privacy protection in real-time surveillance applications," Image and Vision Computing, vol. 94, p. 103859, 2020.

[7] C. Molnar, Interpretable Machine Learning. leanpub.com, 2019. https://christophm.github.io/interpretable-ml-book/.

[8] N. M. Larsen, V. Sigurdsson, and J. Breivik, "The use of observational technology to study in-store behavior: consumer choice, video surveillance, and retail analytics," The Behavior Analyst, vol. 40, no. 2, pp. 343-371, 2017.

[9] K. Ball, "Workplace surveillance: An overview," Labor History, vol. 51, no. 1, pp. 87-106, 2010.

[10] Q. M. Rajpoot and C. D. Jensen, "Video surveillance: Privacy issues and legal compliance," in Promoting Social Change and Democracy Through Information Technology, pp. 69-92, IGI global, 2015.

[11] P. Maheshwari, D. Alex, S. Banerjee, S. Behera, and S. Panda, "Top view person detection and counting for low compute embedded platforms," in Proceedings of the 2018 the 2nd International Conference on Video and Image Processing, pp. 35-43, 2018.

[12] M. Ahmad, I. Ahmed, K. Ullah, I. khan, A. Khattak, and A. Adnan, "Person detection from overhead view: A survey," International Journal of Advanced Computer Science and Applications, vol. 10, no. 4, 2019.

[13] H. H. Ngo, X. Wu, P. D. Le, C. Wilson, and B. Srinivasan, "Dynamic key cryptography and applications.," IJ Network Security, vol. 10, no. 3 , pp. 161-174, 2010.

[14] C.-P. H. Ernst, "Risk hurts fun: The influence of perceived privacy risk on social network site usage," in Factors Driving Social Network Site Usage, pp. 45-56, Springer, 2015.

[15] J. Bhatia and T. D. Breaux, "Empirical measurement of perceived privacy risk," ACM Transactions on Computer-Human Interaction (TOCHI), vol. 25 , no. 6 , pp. 1-47, 2018.

[16] T. Bouwmans and B. Garcia-Garcia, "Background subtraction in real applications: Challenges, current models and future directions," arXiv preprint arXiv:1901.03577, 2019.

[17] M. Piccardi, "Background subtraction techniques: a review," in 2004 IEEE International Conference on Systems, Man and Cybernetics (IEEE Cat. No. 04CH37583), vol. 4, pp. 3099-3104, IEEE, 2004.

[18] C. Cuevas, R. Martínez, and N. García, "Detection of stationary foreground objects: A survey," Computer Vision and Image Understanding, vol. 152, pp. 41-57, 2016.

[19] S. Welsh, A. Hassiotis, G. O'mahoney, and M. Deahl, "Big brother is watching you-the ethical implications of electronic surveillance measures in the elderly with dementia and in adults with learning difficulties," Aging \& mental health, vol. 7, no. 5, pp. 372-375, 2003.

[20] C. Neustaedter, S. Greenberg, and M. Boyle, "Blur filtration fails to preserve privacy for home-based video conferencing," ACM Trans. Comput.-Hum. Interact., vol. 13, p. 1-36, Mar. 2006.

[21] C. Neustaedter and S. Greenberg, "The design of a context-aware home media space for balancing privacy and awareness," in International Conference on Ubiquitous Computing, pp. 297-314, Springer, 2003.

[22] I. Kartika and S. S. Mohamed, "Frame differencing with post-processing techniques for moving object detection in outdoor environment," in 2011 IEEE 7th International Colloquium on Signal Processing and its Applications, pp. 172-176, IEEE, 2011.

[23] J. R. Padilla-López, A. A. Chaaraoui, and F. Flórez-Revuelta, "Visual privacy protection methods: A survey," Expert Systems with Applications, vol. 42, no. 9, pp. 4177-4195, 2015.

[24] B. McPhail, A. Clement, J. Ferenbok, and A. Johnson, “'i'lll be watching you": Awareness, consent, compliance and accountability in video surveillance," in 2013 IEEE International Symposium on Technology and Society (ISTAS): Social Implications of Wearable Computing and Augmediated Reality in Everyday Life, pp. 276-284, IEEE, 2013.

[25] A. Zanotto, N. Conci, and A. Montresor, "Blockchain-grade privacy protection in surveillance systems," in Proceedings of the 13th International Conference on Distributed Smart Cameras, pp. 1-2, 2019. 